Bianca de Souza Rossini Marques

\title{
Desenvolvimento e Certificação por Método Primário de Material de Referência de Condutividade Primária.
}

Dissertação apresentada como requisito parcial para obtenção do título de Mestre pelo Programa de PósGraduação em Metrologia da PUC-Rio. Área de Concentração: Metrologia para Qualidade e Inovação.

Professor Orientador: Prof. José Marcus de Oliveira Godoy

Rio de Janeiro, 28 de Abril de 2010 


\section{Desenvolvimento e Certificação por Método Primário de Material de Referência de Condutividade Primária.}

Dissertação apresentada como requisito parcial para obtenção do título de Mestre pelo Programa de Pós-Graduação em Metrologia para Qualidade Industrial da PUC-Rio. Aprovada pela Comissão Examinadora abaixo assinada.

Prof. José Marcus de Oliveira Godoy

Orientador

Departamento de Química e

Programa de Pós-Graduação em Metrologia Pontifícia Universidade Católica do Rio de Janeiro/PUC-Rio

Isabel Cristina Serta Fraga

Co - Orientadora

INMETRO - Instituto de Metrologia, Normalização e Qualidade Industrial

Vanderléa de Souza

INMETRO - Instituto de Metrologia, Normalização e Qualidade Industrial

Renata Martins Horta Borges

INMETRO - Instituto de Metrologia, Normalização e Qualidade Industrial

Coordenacão Setorial de Pós-graduação:

Prof. José Eugênio Leal

Coordenação Setorial de Pós Graduação do

Centro Tecnológico Científico (PUC-Rio)

Rio de Janeiro, 28 de abril de 2010 
Todos os direitos reservados. É proibida a reprodução total ou parcial do trabalho sem autorização da universidade, da autora e do orientador.

\section{Bianca de Souza Rossini Marques}

Graduou-se em Química pela Universidade do Grande Rio - em 2006. Química do Inmetro no Laboratório de Eletroquímica de 2005 a 2009. Professora de Metrologia do Instituto Federal do Rio de Janeiro desde 2009, ministrando aulas de Qualidade e Normalização.

Ficha Catalográfica

Marques, Bianca de Souza Rossini

Desenvolvimento e certificação por método primário de material de referência de condutividade primária / Bianca de Souza Rossini Marques ; orientador: José Marcus de Oliveira Godoy. - 2009.

96 f. : il. (color.) ; $30 \mathrm{~cm}$

Dissertação (mestrado)-Pontifícia Universidade Católica do Rio de Janeiro, Programa de Pós-Graduação em Metrologia para a Qualidade e Inovação, 2010.

Inclui bibliografia

1. Metrologia - Teses. 2. Material de referência. 3. Certificado. 4. Rastreabilidade. 5. Condutividade eletrolítica. I. Godoy, José Marcus de Oliveira. II. Pontifícia Universidade Católica do Rio de Janeiro. Programa de Pós-Graduação em Metrologia para a Qualidade e Inovação. III. Título.

CDD: 389.1 


\section{Agradecimentos}

Primeiramente a Deus, por tornar possível a realização deste projeto.

A minha mãe que mesmo não estando presente em corpo, lutou a vida inteira pela minha educação, que é a melhor herança que se pode deixar para um filho. E onde quer que ela esteja está feliz por esse momento.

Aos meus avós que sempre estiveram do meu lado e cuidaram de mim.

Ao meu marido Felipe, pelo amor imensurável, pela paciência interminável, e pelo companheirismo e apoio incondicionais.

Ao Prof. José Marcus O. Godoy e a Prof. ${ }^{a}$ Isabel Cristina S. Fraga, pela valiosíssima orientação, pelo estímulo e pelas palavras de coragem nos momentos certos.

Ao Inmetro, pelos auxílios concedidos, sem os quais este trabalho não poderia ter sido realizado.

Aos amigos do Laboratório de Eletroquímica e de Gases do Inmetro, em especial aos amigos Paulo Paschoal Borges, Wiler da Silva Batista Junior e Jéssica Corrêa Lopes, pelo apoio e paciência.

Aos meus colegas da PUC-Rio e a todos os professores e funcionários do Departamento.

Aos professores membros da banca examinadora pela avaliação e contribuição.

A todos os amigos e familiares que acreditaram na realização deste projeto, e cujo carinho e compreensão foram imprescindíveis para sua conclusão.

A todos que direta ou indiretamente contribuíram para que eu pudesse realizar este trabalho. 


\section{Resumo}

De Souza Rossini Marques, Bianca; de Oliveira Godoy, José Marcus. Desenvolvimento e Certificação por Método Primário de Material de Referência Primário de Condutividade Primária. Rio de Janeiro, 2010. 96 p. Dissertação de Mestrado - Programa de Pós-Graduação em Metrologia, Pontifícia Universidade Católica do Rio de Janeiro.

A condutividade eletrolítica é a capacidade de medir o transporte de íons de uma solução. A rastreabilidade é o pré-requisito para a comparabilidade e a uniformidade das medições. No caso das medições de condutividade eletrolítica em soluções, a rastreabilidade é obtida por um sistema primário de condutividade que dará origem aos materiais de referência certificados (MRC) primários. Os MRC são usados para o controle e garantia da qualidade de resultados analíticos, além disso, são essenciais para calibração de instrumentos assegurando a rastreabilidade e confiabilidade dos resultados. A principal motivação para o desenvolvimento deste trabalho é a carência de MRC de baixa condutividade eletrolítica primário devido a sua relevância no controle da pureza da água, matéria-prima para a produção de medicamentos e vacina, além da qualidade do álcool combustível. O objetivo desta dissertação foi desenvolver e certificar um material de referência primário de condutividade eletrolítica de valor nominal de $5 \mu \mathrm{S} \cdot \mathrm{cm}^{-1}$ a $25{ }^{\circ} \mathrm{C}$, produzida a partir do sal $\mathrm{KCl}$ em $30 \%(\mathrm{~m} / \mathrm{m})$ de 1 propanol. Os estudos de homogeneidade, caracterização e estabilidade, foram realizados de acordo com as Normas ISO série 30 . Os resultados obtidos nos estudos de certificação da solução de condutividade eletrolítica, com sua respectiva incerteza expandida foram $(5,00 \pm 0,16) \mu \mathrm{S} \cdot \mathrm{cm}^{-1}$ a $25^{\circ} \mathrm{C}$, com $k=2$, para um nível de confiança de aproximadamente 95\%. A certificação deste material de referência primário contribuirá para a qualidade das medições de condutividade eletrolítica realizadas nos laboratórios do Brasil, o qual irá garantir a rastreabilidade metrológica dos resultados das medições, principalmente no monitoramento da pureza da água e da qualidade do álcool combustível.

\section{Palavras-chave}

Material de Referência Primário; Rastreabilidade; Condutividade Eletrolítica. 


\section{Abstract}

De Souza Rossini Marques, Bianca; de Oliveira Godoy, José Marcus. (Advisor). Development and Certification by the Primary Method of Reference Material of Conductivity Primary. Rio de Janeiro, 2010. 96 p. MSc. Dissertation - Programa de Pós-Graduação em Metrologia, Pontifícia Universidade Católica do Rio de Janeiro.

Certified Reference Material (CRM) is a reference material, accompanied by documentation issued by an authoritative body and providing one or more specified property values with associated uncertainties and traceabilities, using valid procedures. The CRM are used for control and quality assurances of analytical results also are essential to calibrate instruments ensuring the traceability and reliability of results. Electrolytic conductivity is known as the ability of a solution to conduct electrical current. Traceability is a prerequisite for comparability and uniformity of measurements. In the case of electrolytic conductivity measurements on solutions, traceability is obtained through a primary system of conductivity that would lead to the CRM primary electrolytic conductivity. The CRM developed is low conductivity due to its importance in controlling the purity of water, raw material for production of medicines and vaccine, along with analysis of fuel ethanol. The studies of homogeneity, characterization and stability were carried out for CRM $5 \mu \mathrm{S} . \mathrm{cm}^{-1}$ nominal value according to ISO 30 series. The result of studies of certification of the solution of electrolyte conductivity with its corresponding expanded uncertainty, with $k=2$ for a confidence level (CL) of approximately $95 \%$ was $(5.00 \pm 0.16) \mu{\mathrm{S} . \mathrm{cm}^{-1}}^{-}$to $25{ }^{\circ} \mathrm{C}$. The development and certification of primary reference material will contribute to the quality of the results of electrolytic conductivity measurements performed in various laboratories in Brazil and South America, which will guarantee the traceability and reliability of measurement results, especially when is related to monitoring purity of water and analysis of fuel ethanol.

\section{Keywords}

Primary Reference Material; Traceability; Eletrolytic Conductivity. 


\section{Sumário}

1. Introdução 14

1.1. Considerações iniciais 14

1.2. Objetivos geral $\quad 15$

1.3. Objetivos específicos $\quad 15$

1.4. Definição do problema de pesquisa 16

1.5. Motivação 16

$\begin{array}{ll}\text { 1.6. Metodologia } & 16\end{array}$

$\begin{array}{ll}\text { 1.7. Estrutura do trabalho } & 19\end{array}$

2. Fundamentação teórica 20

2.1. A Metrologia 20

2.1.1. Definição e histórico 20

2.1.2. Bureau Internacional de Pesos e Medidas (BIPM) 21

2.1.3. Estrutura da metrologia mundial 23

2.1.4. Confiabilidade metrológica 23

2.1.5. Importância da rastreabilidade na metrologia 24

2.1.6. Instituto Nacional de Metrologia, Normalização e Qualidade Industrial (Inmetro) 25

2.1.7. Acordo de reconhecimento mútuo 27

2.2. A Metrologia Química 28

2.2.1. Rastreabilidade na Metrologia Química 28

2.2.2. A Importância dos Materiais de Referência Certificados para as economias nacionais 31

2.2.3. Code d'Indexation des matériaux de Référence 32

2.2.4. Programa de acreditação de produtores de Materiais de Referência no Brasil 33

2.2.5. Uso de Materiais de Referência Certificados 33

2.2.5.1 Calibração de instrumentos analíticos $\quad 34$

2.2.5.2 Validação de métodos analíticos $\quad 34$

2.2.5.3. Controle de qualidade $\quad 34$

2.2.5.4. Desenvolvimento de Material de Referência Secundário 34

2.3. Características dos Materiais de Referência Certificados 34 
3. Metodologia 36

3.1. Produção e Certificação de Material de Referência 36

3.1.1 Homogeneidade do Material de Referência 38

3.1.2. Estabilidade do Material de Referência 39

3.1.3. Caracterização do Material de Referência 40

3.1.4. Estimativa de incerteza do Material de Referência 41

3.1.5. Certificado e rótulo do Material de Referência 41

3.2. Condutividade Eletrolítica 42

3.2.1. A Rastreabilidade nas medições de Condutividade Eletrolítica 46

3.2.2. A importância de Materiais de Referência Certificados em Condutividade Eletrolítica $\quad 48$

3.2.3. Sistema Primário de Condutividade eletrolítica 49

3.3. Análise de dados 52

3.3.1. Teste de Grubbs 52

3.3.2. Análise de variância 53

3.3.4. Gráficos de controle 53

3.3.5. Teste $F$

3.3.6. Teste $T$

4. Parte experimental 56

4.1. Planejamento e desenvolvimento da solução candidata à Material de Referência Certificado $\quad 56$

4.1.1. Preparação da solução de Condutividade Eletrolítica de $5 \mu \mathrm{S} . \mathrm{cm}^{-1} \quad 56$

4.1.2. Amostragem 57

4.1.3. Procedimento de medição primária de Condutividade Eletrolítica 59

4.1.4. Medições nos estudos de homogeneidade e estabilidade 61

5. Resultados e discussão 63

5.1. Estudo de homogeneidade 63

5.2. Estudo de estabilidade 64

5.3. Caracterização 66

5.4. Estudo de medição de $\mathrm{CO}_{2} \quad 68$

5.5. Correção do resultado de caracterização 69

5.6.1. Incerteza de medição de caracterização $\quad 70$ 
5.6.2. Incerteza de homogeneidade

5.6.3. Estudo de establidade

5.6.4. Estimativa da incerteza final do Material de Referência de $5 \mu \mathrm{S} . \mathrm{cm}^{-1}$

5.6.5. Certificado do Material de Referência de $5 \mu$ S. $\mathrm{cm}^{-1} \quad 74$

5.7. Gráficos de controle $\quad 74$

5.8. Resultado da Comparação Internacional CCQM-P83 75

5.9. Medição de álcool combustível utilizando o Material de Referência Certificado de $5 \mu \mathrm{S} . \mathrm{cm}^{-1} \quad 76$

6. Conclusões e recomendações 80

$\begin{array}{ll}\text { 6.1. Sugestões para futuras pesquisas } & 81\end{array}$

7. Referências Bibliográficas

Anexo A - Valores tabelados para o Teste de Grubbs 87

Anexo B - Valores tabelados distribuição F- probabilidade de 95\% 88

Anexo C - Valores tabelados distribuição T-Student 89

Anexo D - Posição 1 - Estudos de Caracterização 90

$\begin{array}{ll}\text { Anexo E - Posição } 2 \text { - Estudos de Caracterização } & 91\end{array}$

Anexo F - Certificado do MRC de $5 \mu S . \mathrm{cm}^{-1}$ do Inmetro 92

Anexo $\mathrm{G}-$ Certificado do MRC de $5 \mu \mathrm{S} . \mathrm{cm}^{-1}$ da Hamilton 95 


\section{Lista de Figuras}

Figura 1 - Esquema da pesquisa, seus componentes e métodos.

Figura 2 - Esquema dos diversos fatores que contribuem a confiabilidade metrológica em laboratórios de ensaio e calibração [12].

Figura 3 - Hierarquia do Sistema Metrológico.

Figura 4 - Produtos que necessitam da medição de condutividade eletrolítica.

Figura 5 - Célula de condutividade eletrolítica comercial.

Figura 6 - Desenho de uma célula de condutividade eletrolítica comum [34].

Figura 7 - Cadeia de rastreabilidade para medições de condutividade eletrolítica.

Figura 8 - Fotografias ilustrativas da cadeia de rastreabilidade para medições de condutividade eletrolítica.

Figura 9 - Sistema Primário de Medição de Condutividade Eletrolítica do Inmetro.

Figura 10 - Célula Primária de Condutividade Eletrolítica do Inmetro.

Figura 11 - Planilha de dados do Sistema Primário de Medição de Condutividade Eletrolítica.

Figura 12 - Envase da solução de $5 \mu \mathrm{S} \cdot \mathrm{cm}^{-1}$, candidata a MRC.

Figura 13 - Planejamento para a certificação de um MR.

Figura 14 - Tela principal do programa de obtenção de dados do Sistema Primário de Condutividade Eletrolítica.

Figura 15 - Tela de inserção de dados do programa de obtenção de dados do Sistema Primário de Condutividade Eletrolítica.

Figura 16 - Medidor de condutividade eletrolítica utilizado nos estudos de homogeneidade e estabilidade.

Figura 17 - Diagrama de causa e efeito das medições primárias de condutividade eletrolítica.

Figura 18 - Material de Referência certificado de valor nominal de $5 \mu \mathrm{S} . \mathrm{cm}^{-1}$.

Figura 19 - Gráfico de controle de processo de certificação do MR de $5 \mu \mathrm{S} . \mathrm{cm}^{-1}$. 
Figura 20 - Resultado da comparação internacional do CCQM - P83 para medição de $0,5 \mathrm{mS} \cdot \mathrm{m}^{-1}\left(5 \mu \mathrm{S} . \mathrm{cm}^{-1}\right)$. 


\section{Lista de Tabelas}

Tabela 1 - Grandezas e unidades de base do SI [8]. 24

Tabela 2 - Principais diferenças entre os métodos clássico e isócrono. $\quad 40$

Tabela 3 - Diferentes tipos de soluções com seus respectivos valores de condutividade eletrolítica [33].

Tabela 4 - Resultados do estudo de homogeneidade da solução de valor nominal de $5 \mu \mathrm{S} \cdot \mathrm{cm}^{-1}$.

Tabela 5 - Resultados de análise de variância para verificação da homogeneidade da solução de $5 \mu \mathrm{S} \cdot \mathrm{cm}^{-1}$.

Tabela 6 - Resultados das medições do estudo de estabilidade de curta duração a $20{ }^{\circ} \mathrm{C}$ da solução de valor nominal de $5 \mu \mathrm{S} \cdot \mathrm{cm}^{-1}$.

Tabela 7 - Regressão linear para a verificação da estabilidade a $20{ }^{\circ} \mathrm{C}$ da solução de valor nominal de $5 \mu \mathrm{S} \cdot \mathrm{cm}^{-1}$.

Tabela 8 - Resultados das medições do estudo de estabilidade de curta duração a $50 \stackrel{\circ}{\circ}$ da solução de valor nominal de $5 \mu \mathrm{S} \cdot \mathrm{cm}^{-1}$.

Tabela 9 - Regressão linear para a verificação da estabilidade a $50{ }^{\circ} \mathrm{C}$ da solução de valor nominal de $5 \mu \mathrm{S} \cdot \mathrm{cm}^{-1}$.

Tabela 10 - Resultados das medições do estudo de estabilidade de longa duração a $4 \stackrel{\circ}{\circ}$ da solução de valor nominal de $5 \mu \mathrm{S} \cdot \mathrm{cm}^{-1}$.

Tabela 11 - Regressão linear para a verificação da estabilidade a $4 \stackrel{\circ}{ } \mathrm{C}$ da solução de valor nominal de $5 \mu \mathrm{S} \cdot \mathrm{cm}^{-1}$.

Tabela 12 - Resultados da $1^{\text {a }}$ posição de deslocamento da caracterização do candidato a MR.

Tabela 13 - Resultados da $2^{a}$ posição de deslocamento da caracterização do candidato a MR.

Tabela 14 - Resultados do estudo de influência do CO2.

Tabela 15 - Estimativa de incerteza de medição de caracterização da solução de $5 \mu \mathrm{S} \cdot \mathrm{cm}^{-1}$.

Tabela 16 - Resultados da calibração da célula de condutividade do medidor e das condições ambientais realizadas com os MRC do Inmetro e da Hamilton.

Tabela 17 - Resultados das medições de condutividade eletrolítica de álcool combustível. 
Tabela 18 - Resultado do Teste F para comparação do MRC do Inmetro e MRC Hamilton.

Tabela 19 - Resultado do Teste T para comparação do MRC do Inmetro e MRC Hamilton. 